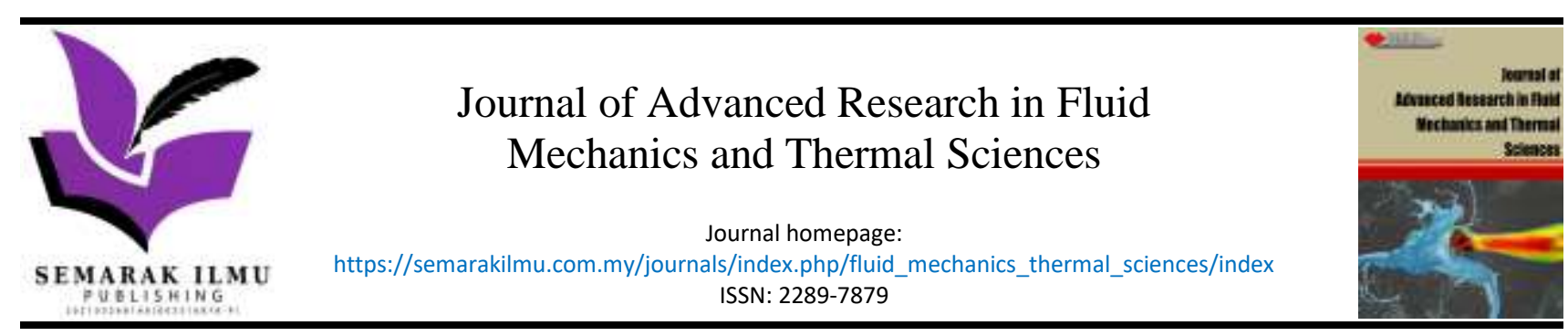

\title{
A Steady Flow of The Viscous Compressible Liquid in Vertical Pipe
}

\author{
Farman Mamedov ${ }^{1,}{ }^{*}$, Nazire Memmedzade ${ }^{1}$ \\ 1 Department of Mathematical Physics Equations, Mathematics and Mechanics Institute of Azerbaijan National Academy of Sciences. \\ B.Vahabzade, 9, AZ 1141, Baku, Azerbaijan
}

\section{ARTICLE INFO}

\section{Article history:}

Received 25 July 2021

Received in revised form 7 November 2021

Accepted 15 November 2021

Available online 4 December 2021

\section{Keywords:}

compressible liquid; steady flow; pressure; velocity distribution

\section{ABSTRACT}

In the study of the flow of gas-liquid mixture over circular vertical pipe rising from the deep zone to ground level, it is observed that, the velocity on surface of tube is much more than in the center of flow. Such a picture is seen also in the water filtration process in sands ordering from high permeability zone to lower. Same phenomena occur in transportation of the water carbon nana-tubes. In order to predict behavior of those processes in this paper, we have studied the compressible liquid flow over the circular vertical pipe ordered from the deep zone to the ground surface for some concrete inlet and outlet regimes of the pipe. The Navier-Stokes equations system as a model of study. For certain inlet and outlet regimes of the flow splitting the equation into the cross section and axes variables, the pressure and velocity distribution are found. The Lane-Emden equation arises for determining the pipe cross section velocity distribution, which is also justified by our calculations on the used model.

\section{Introduction}

There are several physical phenomena in the mass transportation theory which are surprisingly in point of view the classical liquid flow theory. According to the classical theory, the cross-section velocity distribution is a parabola with maximum value at the center of pipe and zero value on the surface of pipe. (Tough several authors are relevant to the power type velocity profiles:

$v=v_{\max }\left[1-\frac{r}{R}\right]^{\frac{1}{n}}$

where the coefficient $\mathrm{n}$ depends on Reynold's number, $v$ max is the maximal velocity [1, 2]). However, in the flow of gas-liquid mixture over circular vertical pipe from the deep zone to the ground level it was observed experimentally that flow velocity on the pipe surface may be much higher than the velocity in center of pipe [3-6]. Such a picture is seen also in the the water filtration process in sands ordered from high permeability zone to lower [7-8], and in the transportation of water over carbon nana tubes [9-12].

\footnotetext{
* Corresponding author.

E-mail address: farman-m@mail.ru

https://doi.org/10.37934/arfmts.89.2.150159
} 
To explain the mentioned experimental results, in this paper, we have considered a flow of compressible liquid over vertical pipe from deep zone to the ground surface. These unfortunately consistently to the particular outlet and and inlet regimes (see, (1)). Also, here it surprises that the well-known Lane-Emden Eq. (2) is raised for determining the velocity distribution on cross section. Earlier such equations arise in the study of astronomy problems, but for the compressible liquid flow, given here, are in the first time. In our studies, we concern mostly to the velocity increase as approaching to the pipe surface. Such a profile as it just was noted is compatible with the water filtration in sands and extraordinary fast transportation of water in carbon nana tubes.

There is a lot of study the one-dimensional flow of compressible viscous liquid in a circular pipe (see, e.g. [13-18]). In those studies, were illustrated different methods for finding the pressure and velocity distribution over the pipe length, where mainly a constant velocity (the average) was taken for the velocity on cross section. Some authors take proportional to velocity term $-\mu v$ in place of the term with Laplace's operator $-\mu \Delta v$ in the Navier-Stokes (NS) equations system in their models (the I.A. Charny equation). In general, a variable velocity over cross section of pipe complicates the splitting of pressure and velocity variables in NS equations system to solve numerically those equations. Because we seek for a concrete outlet $p_{-} 0$ and inlet - $p_{-} 1$ pressure regime to achieve the splitting (see, paragraph 2):

$\ln \frac{p_{1}}{p_{2}}=\frac{\rho_{0} g H}{p_{0}}$

After splitting of the pipe axes $x$ and pipe cross section variables $y, z$ (or) we get the Lane-Emden (LE) of the type to determine the velocity distribution $V=V(y, z)$ over cross section.

$\Delta V+C_{1} V=C_{2} V^{2}$

Here $-H<x<0,0<r<R$, and $\Delta=\frac{\partial^{2}}{\partial y^{2}}+\frac{\partial^{2}}{\partial z^{2}}$ is the Laplace operator. The positive solution of (2) is the subject of this study (the "ground state solution" in the literature, see e.g. [19]). Note, the Lane-Emden equations appear in the different models of mathematical physics and astrophysics [2023], such as, the theory of stellar structure, the thermal behavior of a spherical cloud of gas, isothermal gas spheres or theory of thermionic currents (see, e.g. [24-30]). Those are modeled by the following Lane-Emden equation

$y^{\prime \prime}(x)+\frac{a}{x} y^{\prime}(x)+f(x, y)=g(x), \quad 0 \leq x \leq 1, \quad \alpha \geq 0$

with the Cauchy initial dates $y(0)=a, y^{\wedge}(0)=0$, Many methods have been used to solve this problem (see, e.g. [31-38]). We have taken a linear law of dependence of the density from pressure

$\rho=\frac{\rho_{0}}{p_{0}} p$

\section{Appearance of The LE Equation}

Consider a flow of compressible viscous liquid in the pipe from bottom to top. Assume that, liquid moves parallel to the pipe axis. Locate the $x$ axis parallel to pipe, center it on the top of pipe and order outside to the pipe. So, in our case $x$ is negative. Denote $v(t, x, r)$ the velocity of liquid (ordered 
parallel to $x$ axis) in the point with coordinates $(x, r)$. Here $r$ is the distance from center of cross section; $-x$ is depth on vertical (see, Figure 1 ).

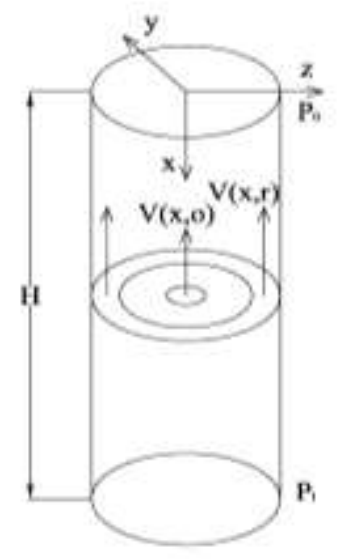

Fig. 1. Flow of compressible liquid oververtical pipe

Denote $p(x)$ the pressure of liquid at the point $x$ (We assume the pressure to be constant over the cross section of pipe). The motion equation of compressible viscous liquid in vertical pipe reads as

$\rho \frac{\partial v}{\partial t}+\rho v \frac{\partial v}{\partial x}+\frac{\partial p}{\partial x}=\mu\left(\frac{\partial^{2} v}{\partial y^{2}}+\frac{\partial^{2} v}{\partial z^{2}}+\frac{\partial^{2} v}{\partial x^{2}}\right)-\rho g$

To obtain a complete system of equations we must to attach (3) the next continuity equation

$\frac{\partial \rho}{\partial t}+\frac{\partial}{\partial x}(\rho v)=0$

Since we consider a steady flow, it holds that $\frac{\partial v}{\partial t}=0$ and $\frac{\partial \rho}{\partial t}=0$, therefore the system of equations (3) and (4) is reduced to the system of

$$
\left\{\begin{aligned}
\rho v \frac{\partial v}{\partial x}+\frac{\partial p}{\partial x}+\rho g & =\mu\left(\frac{\partial^{2} v}{\partial y^{2}}+\frac{\partial^{2} v}{\partial z^{2}}+\frac{\partial^{2} v}{\partial x^{2}}\right) \\
\frac{\partial}{\partial x}(\rho v)=0 & \\
\rho & =\frac{\rho_{0}}{p_{0}} p
\end{aligned}\right.
$$

In order to determine the density, velocity and pressure distribution in the pipe. We search for a solution of (5) the following expressions for velocity and pressure

$p=p_{0}\left(\frac{p_{1}}{p_{0}}\right)^{-\frac{x}{H}}, \quad v=V(r)\left(\frac{p_{1}}{p_{0}}\right)^{\frac{x}{H}}$ 
where $V=V(r)$ is unknown function, $r=\sqrt{y^{2}+z^{2}}, \quad 0 \leq r \leq R, \quad-H<x \leq 0$.

The second condition (5) is fulfilled since the production $\rho v$ does not depend on $x$ :

$\rho v=\frac{\rho_{0}}{p_{0}} \cdot p_{0}\left(\frac{p_{1}}{p_{0}}\right)^{-\frac{x}{H}} \cdot V(r)\left(\frac{p_{1}}{p_{0}}\right)^{\frac{x}{H}}=\rho_{0} V(r)$

Using (6) from (5) it follows

$-\frac{p_{0}}{H}\left(\frac{p_{1}}{p_{0}}\right)^{-\frac{x}{H}} \ln \frac{p_{1}}{p_{0}}+\rho_{0} g\left(\frac{p_{1}}{p_{0}}\right)^{-\frac{x}{H}}+\frac{\rho_{0} V(r)^{2}}{H}\left(\frac{p_{1}}{p_{0}}\right)^{\frac{x}{H}} \ln \frac{p_{1}}{p_{0}}=\mu\left(\frac{p_{1}}{p_{0}}\right)^{\frac{x}{H}}\left(\Delta V+\frac{1}{H^{2}} V(r) \ln ^{2} \frac{p_{1}}{p_{0}}\right)$

Using this, (1), and the expressions for $C_{1}, C_{2}$

$C_{1}=\frac{1}{H^{2}} \ln ^{2} \frac{p_{1}}{p_{0}}, \quad C_{2}=\frac{\rho_{0}}{\mu H} \ln \frac{p_{1}}{p_{0}}$

Hence, we get the next equation

$\frac{\partial^{2} V}{\partial y^{2}}+\frac{\partial^{2} V}{\partial z^{2}}+C_{1} V=C_{2} V^{2}$

for determining the unknown function $\mathrm{V}$ in the ball $B_{R}=\left\{(x, y): x^{2}+y^{2}<R^{2}\right\}$. This is well-known LE equation.

\section{The Darcy-Weisbach Law}

As it follows from the above said content, in order to determine the velocity distribution over cross section of pipe we need to find a solution for the equation (2)

$\frac{\partial^{2} V}{\partial y^{2}}+\frac{\partial^{2} V}{\partial z^{2}}+C_{1} V=C_{2} V^{2} \quad, \quad y^{2}+z^{2}<R^{2}$

under suitable additional boundary conditions. In polar system of coordinates this implies that for the function $V=V(r, \varphi)$ it holds

$\frac{1}{r} \frac{\partial}{\partial r}\left(r \frac{\partial V}{\partial r}\right)+C_{1} V=C_{2} V^{2}$

$0 \leq r<R, 0 \leq \phi<2 \pi$.

We search for a symmetric solution of equation (7). That means, $\partial \mathrm{V} / \partial \mathrm{r}=0$.

There are a lot of numerical techniques to employ on solving of equation (7) (see. e.g. [29, 30]). Also, it is known that the solution of LE equation exists and it is an analytic function of radius $r$ (see, e.g., in [37]). In order to get a unique solution of equation (7) two additional conditions also is needed. One is the symmetry of solution as $\frac{\partial V}{\partial \phi}=0$. Another is the usually taken no-slip condition $V(R)=0$ on the boundary. In the most engineer approximations, the no-slip condition does not hold always in 
reality. Because many authors use the boundary slip condition $\beta \frac{\partial V}{\partial r}+V=0$ in $r=R$ for a second condition.

For a second condition on equation (7) we make use the Darcy-Weisbach law $[3,15,16]$ on friction of liquid with the boundary of pipe in $x=-\frac{H}{2}$. By that law,

$-\left.\frac{\partial p}{\partial x}\right|_{x=-\frac{H}{2}}=\left.f_{c} \frac{\rho \bar{v}^{2}}{4 R}\right|_{x=-\frac{H}{2}}$

Where $\mathrm{f}_{\mathrm{c}}$ is the friction factor, $\left.\bar{v}\right\rfloor_{x=-\frac{H}{2}}$ is an average of velocity over the cross section of pipe in $x=$ $-\frac{H}{2}:$

$\bar{v}\rfloor_{x=-\frac{H}{2}}=\frac{1}{\pi R^{2}} \int_{y^{2}+z^{2}<R^{2}} v\left(-\frac{H}{2}, y, z\right) d y d z=\left(\frac{p_{1}}{p_{0}}\right)^{-\frac{1}{2}} \frac{2}{R^{2}} \int_{0}^{R} r V(r) d r$

From the Eq. (8) and (6) in $x=-\frac{H}{2}$, it follows that

$\frac{p_{0}}{H}\left(\frac{p_{1}}{p_{0}}\right)^{\frac{1}{2}} \ln \frac{p_{1}}{p_{0}}=\left(\frac{p_{1}}{p_{0}}\right)^{-1}\left(\frac{p_{1}}{p_{0}}\right)^{\frac{1}{2}} f_{c} \rho_{0} \frac{\bar{V}^{2}}{4 R}$

Therefore,

$\bar{V}^{2}=\frac{4 p_{1} g R}{f_{c} p_{0}}$

Where

$\bar{V}=\frac{2}{R^{2}} \int_{0}^{R} r V(r) d r$

\section{Approximate Solution of LE Equation}

Since LE is a nonlinear equation, the separation of variables method does not work, 1 and we normally must use a numerical approach, or some asymptotic methods like perturbation method. We have used here the series method.

In reality, $C_{1}$ is very small in comparison with $C_{2}$ in (7):

$\frac{C_{1}}{C_{2}}=\frac{\mu g}{p_{0}} \sim \frac{10^{-4} \cdot 10}{10^{7}}=10^{-10}$

Using it in (7), we may neglect the term $C_{1} \mathrm{~V}$ :

$\frac{1}{r} \frac{\partial}{\partial r}\left(r \frac{\partial V}{\partial r}\right)=C_{2} V^{2}, \quad 0 \leq r \leq R$ 
To find an approximate solution of (7) we set

$V(r)=a_{0}+a_{1} r+a_{2} r^{2}+a_{3} r^{3}+a_{4} r^{4}+\cdots$

Then

$\frac{1}{r}\left(r V^{\prime}(r)\right)^{\prime}=\frac{a_{1}}{r}+4 a_{2}+9 a_{3} r+16 a_{4} r^{2}+25 a_{5} r^{3}+36 a_{6} r^{4}+\cdots$

Also,

$$
V(r)^{2}=a_{0}^{2}+2 a_{0} a_{1} r+\left(a_{1}^{2}+2 a_{0} a_{2}\right) r^{2}+\left(2 a_{1} a_{2}+2 a_{0} a_{3}\right) r^{3}+\left(a_{2}^{2}+2 a_{1} a_{3}+2 a_{0} a_{4}\right) r^{4}+\cdots
$$

Inserting last equations in (7), we get

$$
\begin{gathered}
\frac{a_{1}}{r}+4 a_{2}+9 a_{3} r+16 a_{4} r^{2}+25 a_{5} r^{3}+36 a_{6} r^{4}+\cdots=C_{2} a_{0}^{2}+2 C_{2} a_{0} a_{1} r+C_{2}\left(a_{1}^{2}+2 a_{0} a_{2}\right) r^{2} \\
+C_{2}\left(2 a_{1} a_{2}+2 a_{0} a_{3}\right) r^{3}+C_{2}\left(a_{2}^{2}+2 a_{1} a_{3}+2 a_{0} a_{4}\right) r^{4}+\cdots
\end{gathered}
$$

Equating the terms with same powers of $r$ from there we infer

$$
\left\{\begin{array}{c}
a_{1}=0 \\
4 a_{2}=C_{2} a_{0}^{2} \\
9 a_{3}=C_{2} a_{0} a_{1} \\
16 a_{4}=C_{2}\left(a_{1}^{2}+2 a_{0} a_{2}\right) \\
25 a_{5}=C_{2}\left(2 a_{1} a_{2}+2 a_{0} a_{3}\right) \\
36 a_{6}=C_{2}\left(a_{2}^{2}+2 a_{1} a_{3}+2 a_{0} a_{4}\right) \\
\ldots \quad \ldots \quad \ldots
\end{array}\right.
$$

Therefore,

$$
\left\{\begin{array}{c}
a_{1}=0 \\
4 a_{2}=C_{2} a_{0}^{2} \\
a_{3}=0 \\
16 a_{4}=2 C_{2} a_{0} a_{2} \\
a_{5}=0 \\
36 a_{6}=C_{2}\left(a_{2}^{2}+2 a_{0} a_{4}\right) \\
\ldots \quad \ldots \quad \ldots
\end{array}\right.
$$

In order to determine the coefficients of series (10) uniquely we have to attach one equation else to the system (11). We derive it from (9).

Determine $V$ from expression (10):

$\bar{V}=a_{0}+\frac{2}{3} a_{1} R+\frac{2}{4} a_{2} R^{2}+\frac{2}{5} a_{3} R^{3}+\frac{2}{6} a_{4} R^{4}+\ldots$

therefore, 


$$
\begin{gathered}
\bar{V}^{2}=a_{0}^{2}+\frac{4}{3} a_{0} a_{1} R+\left(a_{0} a_{2}+\frac{4}{9} a_{1}^{2}\right) R^{2}+\left(\frac{2}{3} a_{1} a_{2}+\frac{4}{5} a_{0} a_{3}\right) R^{3}+\left(\frac{2}{3} a_{0} a_{4}+\frac{1}{4} a_{2}^{2}+\frac{8}{15} a_{1} a_{3}\right) R^{4} \\
+\ldots
\end{gathered}
$$

Therefore, from (12), and since $a_{1}=a_{3}=a_{5}=0$, we get

$\frac{4 g R p_{1}}{p_{0} f_{c}}=a_{0}^{2}+a_{0} a_{2} R^{2}+\left(\frac{2}{3} a_{0} a_{4}+\frac{1}{4} a_{2}^{2}\right) R^{4}+\cdots$

Gathering (11) and (13) together we get a number system of equations for the unknowns $a_{0}, a_{2}, a_{4}, \ldots, a_{6}, \ldots$ as following

$$
\left\{\begin{array}{c}
C_{2}=\frac{\rho_{0}^{2} g}{\mu p_{0}} \\
\frac{4 g R p_{1}}{p_{0} f_{c}}=a_{0}^{2}+a_{0} a_{2} R^{2}+\ldots \\
4 a_{2}=C_{2} a_{0}^{2} \\
16 a_{2}=2 C_{2} a_{0} a_{2} \\
36 a_{6}=C_{2}\left(a_{2}^{2}+2 a_{0} a_{4}\right) \\
\ldots
\end{array}\right.
$$

The approximate solution of (14) is located from the system

$\frac{\rho_{0}^{2} g R^{2}}{4 \mu p_{0}} a_{0}^{3}+a_{0}^{2}=\frac{4 g R p_{1}}{p_{0} f_{c}}$

Therefore, we get the cubic equation for unknown ao

$a_{0}^{3}+\frac{4 \mu p_{0}}{\rho_{0}^{2} g R^{2}} a_{0}^{2}-\frac{16 \mu p_{1}}{\rho_{0}^{2} R f_{c}}=0$

Present it in the form

$A x^{3}+B x^{2}+C_{x}+D=0$

This equation has one real and two complex or three real roots concerning unknown a . Inserting here $a=\frac{B}{A}, b=\frac{C}{A}, c=\frac{D}{A}$ we get

$x^{3}+a x^{2}+b x+c=0$

Denote

$p=b-\frac{a^{2}}{3}, \quad q=\frac{2 a^{3}}{27}-\frac{a b}{3}+c, \quad D=\frac{q^{2}}{4}+\frac{p^{3}}{27}$

If $D>0$ then the cubic equation (15) has one real and two complex solutions. If $D<0$ the (15) have three distinct real solutions. 
In our case of equation (4) we use the computer calculations (see e.g. www.1728.org/cubic.htm) and the coefficients are $A=1, B=\frac{4 \mu p_{0}}{\rho_{0}^{2} g R^{2}}, C=0, D=-\frac{16 \mu p_{1}}{\rho_{0}^{2} R f_{c}} A=1$. The value of reasonable root gives $a_{0}$ ( i.e. $x_{1}=a_{0}$ ) and $a_{2}=\frac{a_{0}^{2} \rho_{0}^{2} g}{4 \mu p_{0}}, a_{4}=\frac{\rho_{0}^{2} g}{8 \mu p_{0}} a_{0} a_{2}, \ldots$. Notice, in our considerations, the value of $p_{1}$ is associate with $p_{0}$ by the formula

$p_{1}=p_{0} e^{\frac{\rho_{0} g H}{p_{0}}}$

Inserting the funded values $a_{0}, a_{2}, a_{4}, \ldots, a_{6}$ into the expansion (10), we get the velocity distribution over cross section of the well. A further using of expression for cross section velocity profile $V(r)$ into (6) we finally, find the pressure, density, and velocity distribution low in the pipe.

\section{Results}

We study the velocity and pressure distribution in the one-dimensional flow of compressible viscous liquid in vertical pipe from bottom to top. A variable velocity over cross section of the pipe is considered. Also, to take into account boundary friction of the liquid with pipe wall the DarcyWeisbach law is used. Truthfulness of our approach justified with successfully splitting of the variables in solving process of NS equations system: one is the Lane-Emden equation over cross section of pipe, another is an ODE over the well axis. Really, we have restricted on the particular depression as the (1), but it is justified by the fact that, an exact solution is found.

\section{Conclusions}

In many real well conditions, the oil, gas and water mixture move from the depth of the well to the ground surface over the vertical pipe. This is a mixture which obeys the compressible liquid ability. Its viscosity looks like the gas viscosity. Also, the velocity distribution in the cross section of pipe is not constant in reality. Our considerations keep this reality, taking the variable velocity profile, even increasing from center to the boundary of pipe. The profile with zero boundary velocity is not suitable, in this case. We take the Darcy-Weisbach law in place, in order to take into the account, the friction of liquid with boundary of pipe. Applying splitting allows to find the exact solution of NS equations system, and therefore to determine the velocity, density and pressure distribution in pipe. Along the way, we came to the LE equation, which earlier arose in problems of astronomy. Also, these results open a new view to the LE equation in the sense of applications.

\section{References}

[1] Munson, Bruce R., Donald F. Young, and Theodore H. Okiishi. "Fundamentals of fluid mechanics." Oceanographic Literature Review 10, no. 42 (1995): 831.

[2] Pope, S. B. "Turbulent Flows, Fifth printing." (2008).

[3] Manning, Francis, and Richard Thompson. Oilfield processing of petroleum: natural gas. PennWell Books, LLC, 1991.

[4] Štigler, Jaroslav. "Analytical velocity profile in tube for laminar and turbulent flow." Engineering Mechanics 21 , no. 6 (2014): 371-379. https://doi.org/10.1002/ihbp.66

[5] Loilier, Pauline. "Numerical simulation of two-phase gas-liquid flows in inclined and vertical pipelines." PhD diss., Cranfield University, 2006.

[6] Hatta, Natsuo, Hitoshi Fujimoto, Makoto Isobe, and Jung-Seock Kang. "Theoretical analysis of flow characteristics of multiphase mixtures in a vertical pipe." International Journal of Multiphase Flow 24, no. 4 (1998): 539-561. https://doi.org/10.1016/S0301-9322(97)00074-8 
[7] Alabi, O. O., D. T. Ajah, and L. K. Abidoye. "Mathematical Modeling of Hydraulic Conductivity in Homogeneous Porous Media: Influence of Porosity and Implications in Subsurface Transport of Contaminants." Electronic Journal of Geotechnical Engineering 21, no. 1 (2016): 89.

[8] Cedergren, Harry R. Seepage, drainage, and flow nets. Vol. 16. John Wiley \& Sons, 1997.

[9] Joseph, Sony, and N. R. Aluru. "Why are carbon nanotubes fast transporters of water?." Nano letters 8, no. 2 (2008): 452-458. https://doi.org/10.1021/nl072385q

[10] Whitby, Max, Laurent Cagnon, Maya Thanou, and Nick Quirke. "Enhanced fluid flow through nanoscale carbon pipes." Nano letters 8, no. 9 (2008): 2632-2637. https://doi.org/10.1021/nl080705f

[11] Halim, Nur Fazlin Che, and Nor Azwadi Che Sidik. "Mixing Chamber for Preparation of Nanorefrigerant." Journal of Advanced Research in Applied Sciences and Engineering Technology 21, no. 1 (2020): 32-40. https://doi.org/10.37934/araset.21.1.3240

[12] Mahat, Rahimah, Sharidan Shafie, and Fatihhi Januddi. "Numerical Analysis of Mixed Convection Flow Past a Symmetric Cylinder with Viscous Dissipation in Viscoelastic Nanofluid." CFD Letters 13, no. 2 (2021): 12-28.

[13] Muskat, M The flow of homogenous fluids through porous media, T.W. Edwards inc ann ARBOR, Michigan, 1946.

[14] Wang, C. Y. "Exact solutions of the steady-state Navier-Stokes equations." Annual Review of Fluid Mechanics 23, no. 1 (1991): 159-177. https://doi.org/10.1146/annurev.fl.23.010191.001111

[15] Çengel, Yunus A., Robert H. Turner, John M. Cimbala, and Mehmet Kanoglu. Fundamentals of thermal-fluid sciences. Vol. 703. New York: McGraw-Hill, 2008.

[16] White, Frank M. "Fluid Mechanics, McGraw-Hill." New York (1994).

[17] Basniev, K. S., A. M. Vlasov, I. N. Kochina, and V. M. Maksimov. "Underground Hydraulics [in Russian]." (1986).

[18] I.A. Charny, Underground Hydrodynamics, Gostopizdat, Moscow, 1963, p 396.

[19] Lions, H. Berestycki-PL. "Nonlinear scalar field equations, I. Existence of a ground state." Arch. Rational Mech. Anal 82, no. 4 (1983): 313-345. https://doi.org/10.1007/BF00250555

[20] Abd-Elhameed, Waleed M., Youssri Youssri, and Eid H. Doha. "New solutions for singular Lane-Emden equations arising in astrophysics based on shifted ultraspherical operational matrices of derivatives." Computational Methods for Differential Equations 2, no. 3 (2014): 171-185.

[21] Fowler, R. H. "Emden's equation: The solutions of Emden's and similar differential equations." Monthly Notices of the Royal Astronomical Society 91 (1930): 63. https://doi.org/10.1093/mnras/91.1.63

[22] Singh, Om P., Rajesh K. Pandey, and Vineet K. Singh. "An analytic algorithm of Lane-Emden type equations arising in astrophysics using modified homotopy analysis method." Computer Physics Communications 180, no. 7 (2009): 1116-1124. https://doi.org/10.1016/j.cpc.2009.01.012

[23] Dehghan, Mehdi, and Fatemeh Shakeri. "Approximate solution of a differential equation arising in astrophysics using the variational iteration method." New Astronomy 13, no. 1 (2008): 53-59. https://doi.org/10.1016/i.newast.2007.06.012

[24] J Chandrasekhar, Subrahmanyan, and Subrahmanyan Chandrasekhar. An introduction to the study of stellar structure. Vol. 2. Courier Corporation, 1957.

[25] Richardson, Owen Willans. The emission of electricity from hot bodies. Vol. 4. Longmans, Green and Company, 1921.

[26] Bellahcene, Lahcene, Djamel Sahel, and Aissa Yousfi. "Numerical study of shell and tube heat exchanger performance enhancement using nanofluids and baffling technique." Journal of Advanced Research in Fluid Mechanics and Thermal Sciences 80, no. 2 (2021): 42-55. https://doi.org/10.37934/arfmts.80.2.4255

[27] Amanov, Rabil A., and Farman Imran Mamedov. "Regularity of the solutions of degenerate elliptic equations in divergent form." Mathematical Notes 83, no. 1 (2008): 3-13. https://doi.org/10.1134/S000143460801001X

[28] Aziz, Wan Nor Suhaila Wan, Shahrul Kadri Ayop, and Rosazley Ramly. "Temperature and Concentration Dependent Viscosity of Microcrystalline Cellulose in Water." Journal of Advanced Research in Fluid Mechanics and Thermal Sciences 80, no. 2 (2021): 74-81. https://doi.org/10.37934/arfmts.80.2.7481

[29] Dixon, J. M., and J. A. Tuszyński. "Solutions of a generalized Emden equation and their physical significance." Physical Review A 41, no. 8 (1990): 4166. https://doi.org/10.1103/PhysRevA.41.4166

[30] Parand, K., Mehdi Dehghan, A. R. Rezaei, and S. M. Ghaderi. "An approximation algorithm for the solution of the nonlinear Lane-Emden type equations arising in astrophysics using Hermite functions collocation method." Computer Physics Communications 181, no. $6 \quad$ (2010): $1096-1108$. https://doi.org/10.1016/j.cpc.2010.02.018

[31] Ertürk, Vedat Suat. "Differential transformation method for solving differential equations of Lane-Emden type." Mathematical and computational Applications 12, no. $3 \quad$ (2007): 135-139. https://doi.org/10.3390/mca12030135

[32] Khalique, C. M., and P. Ntsime. "Exact solutions of the Lane-Emden-type equation." New Astronomy 13, no. 7 (2008): 476-480. https://doi.org/10.1016/j.newast.2008.01.002 
[33] Lin, Huei-li, and Weichung Wang. "A Palais-Smale approach to Lane-Emden equations." Journal of mathematical analysis and applications 330, no. 2 (2007): 1220-1237. https://doi.org/10.1016/i.jmaa.2006.08.023

[34] Weisstein, Eric W. " Lane-Emden Differential Equation." https://mathworld. wolfram. com/ (2004).

[35] Momoniat, E., and C. Harley. "Approximate implicit solution of a Lane-Emden equation." New Astronomy 11, no. 7 (2006): 520-526. https://doi.org/10.1016/j.newast.2006.02.004

[36] Ramos, J. I. "Series approach to the Lane-Emden equation and comparison with the homotopy perturbation method." Chaos, Solitons \& Fractals 38, no. 2 (2008): 400-408. https://doi.org/10.1016/i.chaos.2006.11.018

[37] Shawagfeh, N. T. "Nonperturbative approximate solution for Lane-Emden equation." Journal of Mathematical Physics 34, no. 9 (1993): 4364-4369. https://doi.org/10.1063/1.530005

[38] Wazwaz, Abdul-Majid. "A new algorithm for solving differential equations of Lane-Emden type." Applied mathematics and computation 118, no. 2-3 (2001): 287-310. https://doi.org/10.1016/S0096-3003(99)00223-4 